\title{
LE COMPORTEMENT DE REPRODUCTION DU GOUJON, GOBIO GOBIO, EN AQUARIUM : PREMIERS RÉSULTATS.
}

\author{
P. PONCIN (1), J. JEANDARME (1), J. RINCHARD (2) et P. KESTEMONT (2)
}

(1) Université de Liège, Service d'Ethologie et de Psychologie animale, Laboratoire d'Ethologie des Poissons, 22 quai Van Beneden, B-4020 Liège, Belgique.

(2) Facultés Universitaires Notre-Dame de la Paix, Unité de Recherche en Biologie des Organismes, Laboratoire d'Ecologie des Eaux Douces, 61 rue de Bruxelles, B-5000 Namur, Belgique.

\section{RÉSUMÉ}

Le comportement de reproduction du goujon Gobio gobio L. a été analysé à partir de 15 séquences complètes d'accouplement d'une même femelle en aquarium. L'accouplement implique un mâle et une femelle qui effectuent une nage ondulatoire vers la surface de l'eau où les ovules sont libérés. Des mâles opportunistes peuvent parfois participer à cette séquence. L'évolution des comportements non sexuels (nage, fouille, montée en surface) et sexuels (nage de synchronisation, accouplement) sur une période de 14 heures est aussi décrite.

\section{PRELIMINARY RESULTS ON THE SPAWNING BEHAVIOUR OF THE GUDGEON, GOBIO GOBIO, IN AQUARIUM.}

\section{ABSTRACT}

This study describes the reproductive behaviour of the gudgeon Gobio gobio L. in aquarium. 15 spawning sequences are analysed. Each one involves one male and one female undulating rapidly towards the water surface where eggs and sperm are released. Some sneaking males are sometimes observed participating in spawning acts. The reproductive (synchronous swimming, spawning attempts) and non-reproductive (swimming, foraging, surfacing) behaviours are also recorded and described for a 14 hours period.

\section{INTRODUCTION}

Le comportement de reproduction et les tactiques d'accouplement des poissons européens d'eau douce ont déjà été décrits en détail chez bon nombre d'espèces (voir PONCIN, 1996, pour synthèse). En ce qui concerne le goujon (Gobio gobio Linné, 1766), sa reproduction a déjà fait l'objet d'études qui en abordent les aspects démographiques (âge et croissance des géniteurs, fécondité) et écologiques (dates et températures de 
ponte, pontes fractionnées, développement embryonnaire) (LOBON-CERVIA et TORRES, 1984 ; MANN, 1980 ; PENAZ et PROKES, 1978). Complétant ces recherches de terrain, plusieurs travaux décrivent les modifications saisonnières dans l'histologie des ovaires (KESTEMONT, 1987) et des testicules (KESTEMONT, 1989) et mettent en évidence l'influence des facteurs environnementaux (photopériode, température) sur la dynamique de l'ovogenèse (KESTEMONT, 1990) et de la spermatogenèse (KESTEMONT, 1988 a). La production d'ovules fécondables par insémination artificielle a également fait l'objet d'une attention particulière (KESTEMONT, 1988 b). L'intérêt du goujon pour la pisciculture (HOESTLAND et BRUNET, 1973) a permis le développement de techniques de production intensive qui impliquent la reproduction naturelle contrôlée (KESTEMONT et MELARD, 1994), bien que le comportement sexuel du goujon n'ait jamais été décrit. En effet, la petite taille du poisson, l'absence de dimorphisme sexuel marqué et la rapidité des accouplements rendent très difficiles les observations en milieu naturel mais aussi en captivité (étangs, bassins d'élevage). Cette étude préliminaire présente une description détaillée des parades sexuelles chez des individus maintenus en aquarium et filmés à l'aide d'un équipement vidéo approprié.

\section{MATÉRIEL ET MÉTHODES}

Les goujons ont soit été capturés par pêche à l'électricité dans la Vesdre (région de Liège, Belgique), soit été produits en pisciculture dans les installations expérimentales des Facultés Universitaires Notre-Dame de la Paix de Namur. Les individus matures ont été sélectionnés pour les expériences qui sont réalisées pendant la période de reproduction naturelle. Les femelles ( $n=22$ ind. sauvages et 10 ind. d'élevage) et les mâles ( $n=25$ ind. sauvages et 10 ind. d'élevage) mesuraient en moyenne respectivement $133 \pm 13 \mathrm{~mm}$ et $128 \pm 10 \mathrm{~mm}$ (longueur à la fourche).

Les goujons étaient maintenus dans des aquariums de 500 I $(1,8 \times 0,6 \times 0,5 \mathrm{~m})$ possédant un système de filtration indépendant. Un courant d'eau était créé dans chaque aquarium ( $\pm 21 \mathrm{~cm} \mathrm{sec}^{-1}$ au milieu des aquariums) au moyen d'une pompe. La température de l'eau était comprise entre 22 et $24^{\circ} \mathrm{C}$ et la photopériode fixée à $16 \mathrm{~L} / 8 \mathrm{~N}$. Les goujons étaient nourris à satiété au moyen de larves de chironomide surgelées (vers de vase). Afin de procurer aux poissons une zone d'ombrage, chaque aquarium était muni d'un abri en schistes ( $\pm 1000 \mathrm{~cm}^{2} ; \pm 1 / 10$ de l'aquarium) suspendu à $20 \mathrm{~cm}$ du fond. Ce dernier était tapissé de fins graviers pouvant être considérés comme un substrat de ponte favorable à la reproduction du goujon (espèce psammophile qui pond sur des fonds sableux; BALON, 1975 ; PHILIPPART, 1989). Une zone de cailloux ( $\pm 1 / 10$ de l'aquarium) était aussi aménagée sur le fond afin de diversifier le substrat (voir figure 2).

Les observations comportementales ont été enregistrées à l'aide d'une caméra couleur et d'un magnétoscope, commandés à partir d'un local adjacent au laboratoire (PONCIN et RUWET, 1996).

Les individus mûrs étaient sélectionnés sur la base de critères morphologiques (papille génitale saillante, abdomen dilaté chez les femelles; présence de sperme chez les mâles) et traités dès la première semaine de leur transfert en aquarium. Les expériences ont été menées sur 8 groupes comportant de 1 à 5 femelles et de 3 à 5 mâles. Afin de stimuler l'activité reproductrice des poissons et d'induire la ponte, mâles et femelles ont reçu une seule injection d'extraits hypophysaires de carpe (10 ug g ${ }^{-1}$ de poids vif) suivant la méthode décrite par KESTEMONT (1988 b). Les femelles étaient marquées par une légère ablation au niveau de la nageoire caudale. Les injections étaient administrées vers $\mathrm{OHOO}$ (heure locale). 
Les données étaient ensuite enregistrées dès l'aube $(\mathrm{TO}=$ allumage de l'éclairage $=6 \mathrm{H} 00$ ) à raison de 15 minutes toutes les heures, jusqu'à disparition totale des comportements reproducteurs (soit sur une période de 14 heures). Les bandes vidéo ont été visionnées image par image pour la déscription des accouplements. Les comportements ont ensuite été quantifiés en fréquence ou en durée au moyen d'un programme informatique ad hoc (programme " FERRARA ", Faculté de Psychologie, ULg).

\section{RÉSULTATS}

\section{Description du comportement sexuel}

L'éthogramme du goujon regroupe des comportements non sexuels comme la nage, le repos; la fouille dans le gravier, la montée en surface, et des comportements sexuels comme la nage de synchronisation qui consiste en une nage très rapide de deux individus côte à côte, ou l'accouplement, décrit ci-dessous.

Sur les 32 femelles hypophysées, une seule d'origine sauvage (dans un groupe composé de 3 femelles et 4 mâles) a développé des séquences comportementales complètes aboutissant à l'enregistrement et à l'analyse de 15 actes de ponte dont la plupart avec une libération d'ovules bien visibles. Des séquences incomplètes ont été relevées chez les femelles de 2 autres groupes sans qu'elles aboutissent à la pónte.

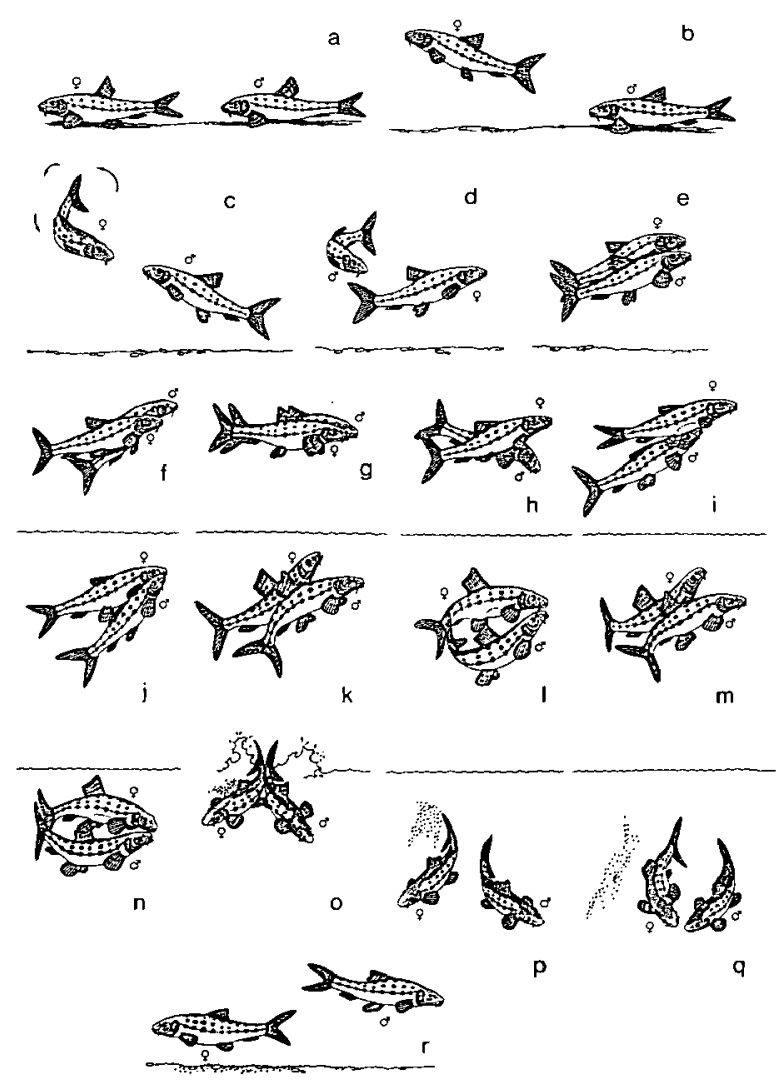

Figure 1

Séquence-type de reproduction d'un mâle et d'une femelle goujon en aquarium. Dessin de A.M. MASSIN.

Figure 1

Spawning sequence of a male and a female gudgeon in aquarium. Drawing by A.M. MASSIN. 
La séquence-type d'accouplement débute par l'activation de la femelle qui nage en tournoyant vers la surface de l'eau (fig. $1 \mathrm{a}, \mathrm{b}, \mathrm{c}$ ). Elle est aussitôt suivie par un mâle qui se place à ses côtés, puis synchronise sa nage avec celle de la femelle (fig. $1 \mathrm{~d}, \mathrm{e}, \mathrm{f}$ ). Parfois, c'est le mâle qui prend l'initiative de nager vers la surface. II passe ensuite plusieurs fois sous l'abdomen de la femelle, en la stimulant par des petits coups de tête (fig. $1 \mathrm{~g}, \mathrm{~h}$, i). II donne alors un brusque coup de nez au niveau de la tête de la femelle, ce qui déclenche la montée rapide du couple vers la surface (fig. $1 \mathrm{j}$ ). Chaque individu forme alors un arc de cercle, alternativement concave ou convexe (fig. $1 \mathrm{k}, \mathrm{I}, \mathrm{m}, \mathrm{n}$ ). La femelle frappe ensuite la surface de l'eau avec sa queue et libère ses ovules. Au même moment, le mâle projette son pédoncule caudal sur celui de la femelle et libère très vraisemblablement son sperme à ce moment (fig. $1 \mathrm{o}, \mathrm{p}$ ). Ce comportement peut aussi être observé sans que les individus percent la surface de l'eau (fig. 2). Le couple se dissocie ensuite, chacun reprenant sa place sur le fond de l'aquarium (fig. 1 q, r). Les œufs, entraînés par le courant d'eau, se déposent sur le substrat où ils adhèrent, avant d'être totalement ou en partie consommés par les reprod!ucteurs eux-mêmes ou des goujons qui ne participent pas aux accouplements. Sur les 15 séquences de ponte analysées, la femelle s'est accouplée 10 fois avec 1 seul mâle. Dans les autres cas, bien que la séquence comportementale était initiée par un seul mâle, elle se poursuivait avec $1(2 x)$ ou $2(3 x)$ individus supplémentaires, opportunistes, qui se positionnaient aux côtés de la femelle lorsqu'elle émettait ses ovules.

\section{Evolution du comportement au cours du temps}

La figure 3 illustre l'évolution des 5 principaux comportements des 4 mâles goujons et de la femelle mûre, depuis l'allumage de l'éclairage $(6 \mathrm{HOO})$ jusqu'à l'achèvement des pontes (19H00). On note que l'activité (nage, fouille du gravier) augmente progressivement jusqu'à la période d'accouplement, puis s'estompe. La recherche de nourriture (fouille du gravier) est plus importante chez les mâles que chez les femelles. Ils se nourrissent principalement des œufs fraîchement pondus par la femelle ! Les premiers accouplements sont observés environ 10 heures (soit 230 degrés-heure) après l'injection hormonale et s'étalent pendant environ 7 heures. On note toutefois une diminution d'activité vers $12 \mathrm{H} 00$. Durant la période d'accouplements, on relève de nombreuses nages de synchronisation entre mâle et femelle, ainsi que d'abondantes montées en surface.

\section{DISCUSSION ET CONCLUSION}

Cette étude décrit le comportement de reproduction du goujon, méconnu jusqu'alors. Celui-ci se déroule principalement en couple, la libération des ovules par la femelle nécessitant plusieurs mouvements d'incurvation latérale préalables. D'autres mâles opportunistes peuvent tenter de participer à la fertilisation des ovules. Si nos observations sont confirmées, le mode de reproduction du goujon pourrait être considéré comme polygame, chaque femelle étant susceptible de se reproduire avec plusieurs mâles successivement, voire même simultanément, et chaque mâle pouvant se reproduire successivement avec des femelles différentes (voir TURNER, 1986, pour définition de la polygamie). Etant donné la difficulté d'observation du sperme émis par les mâles, seule une analyse de la paternité des œufs ou des larves, après la fécondation, pourrait nous renseigner sur la participation effective de chacun à la descendance. Cette recherche est actuellement en cours chez le barbeau fluviatile Barbus barbus et le barbeau méridional $B$. meridionalis (PONCIN et al., 1995). Au même titre que ces dernières espèces, le goujon ne semble pas présenter de comportements agressifs (entre mâles ou entre mâles et femelles) associés à la reproduction. Toutefois, des nages de synchronisation (appelées nages de front chez le barbeau, car les individus nagent tête contre tête) peuvent aussi être observées. Etant donné que le comportement sexuel du goujon n'a pas été observé chez des individus non hypophysés, 

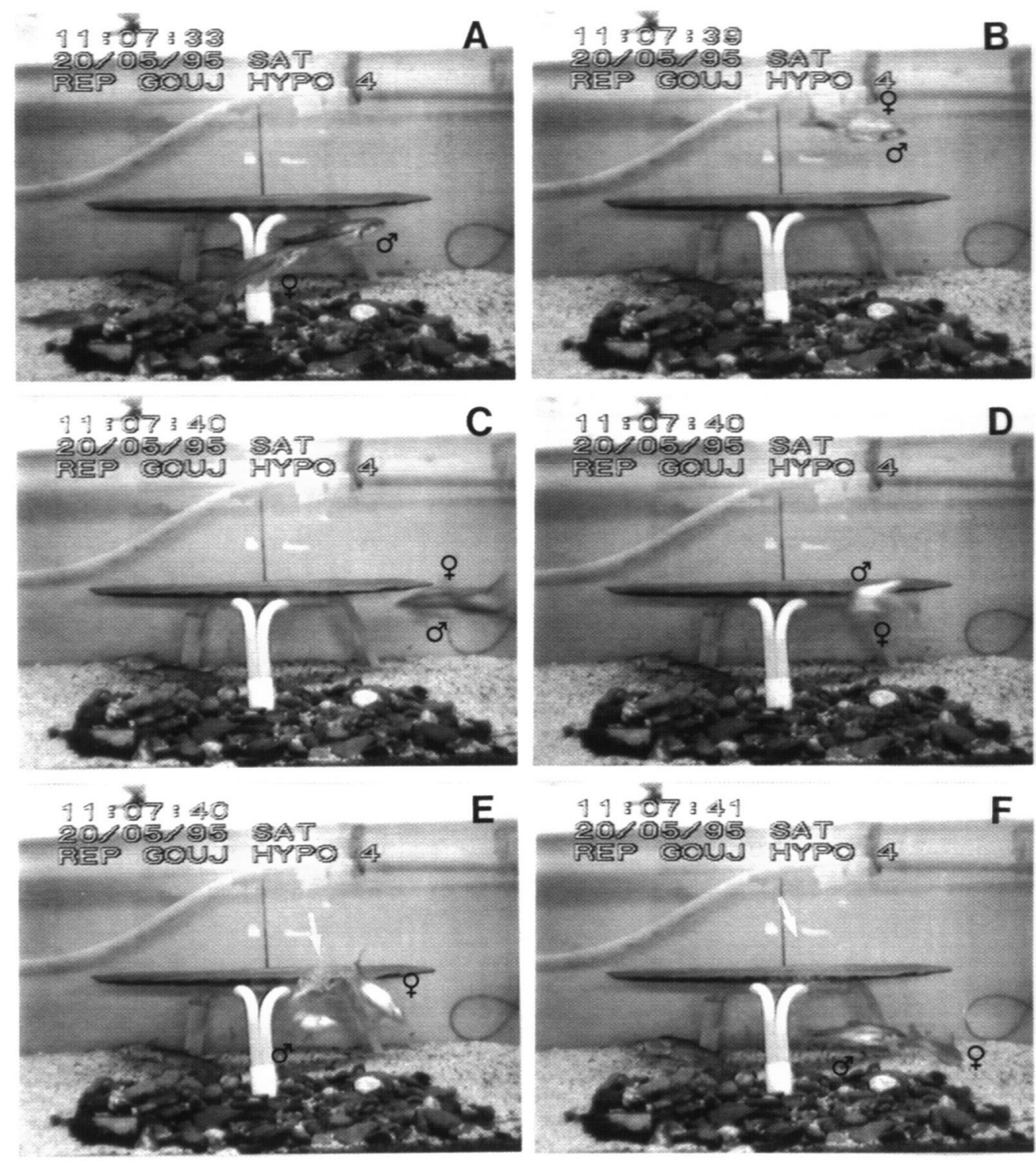

Figure 2

Principaux comportements sexuels du goujon en aquarium lors d'une séquence où les individus ne percent pas la surface de l'eau.

A : le mâle monte vers la surface suivi par la femelle;

$B$ et $C$ : le mâle et la femelle nagent rapidement en se synchronisant ;

$D$ et $E$ : accouplement : les deux individus projettent leurs pédoncules caudaux l'un contre l'autre et expulsent leurs produits sexuels. En " $E$ " et " $F$ ", les œufs sont indiqués par des flèches ;

F : séparation du couple et éparpillement des œufs sur le substrat.

\section{Figure 2}

Main sexual behaviours in gudgeon in aquarium during a sequence in which fish do not break the surface.

$A$ : the male and the female swim towards the water"surface ;

$B$ and $C$ : synchronous swimming;

$D$ and $E$ : spawning : the male and the female join their caudal fins and release eggs and sperm. In « $E$ " and " $F$ ", the arrows indicate the released eggs;

$F$ : separation of the spawning pair and scatering of the eggs on the substrate. 

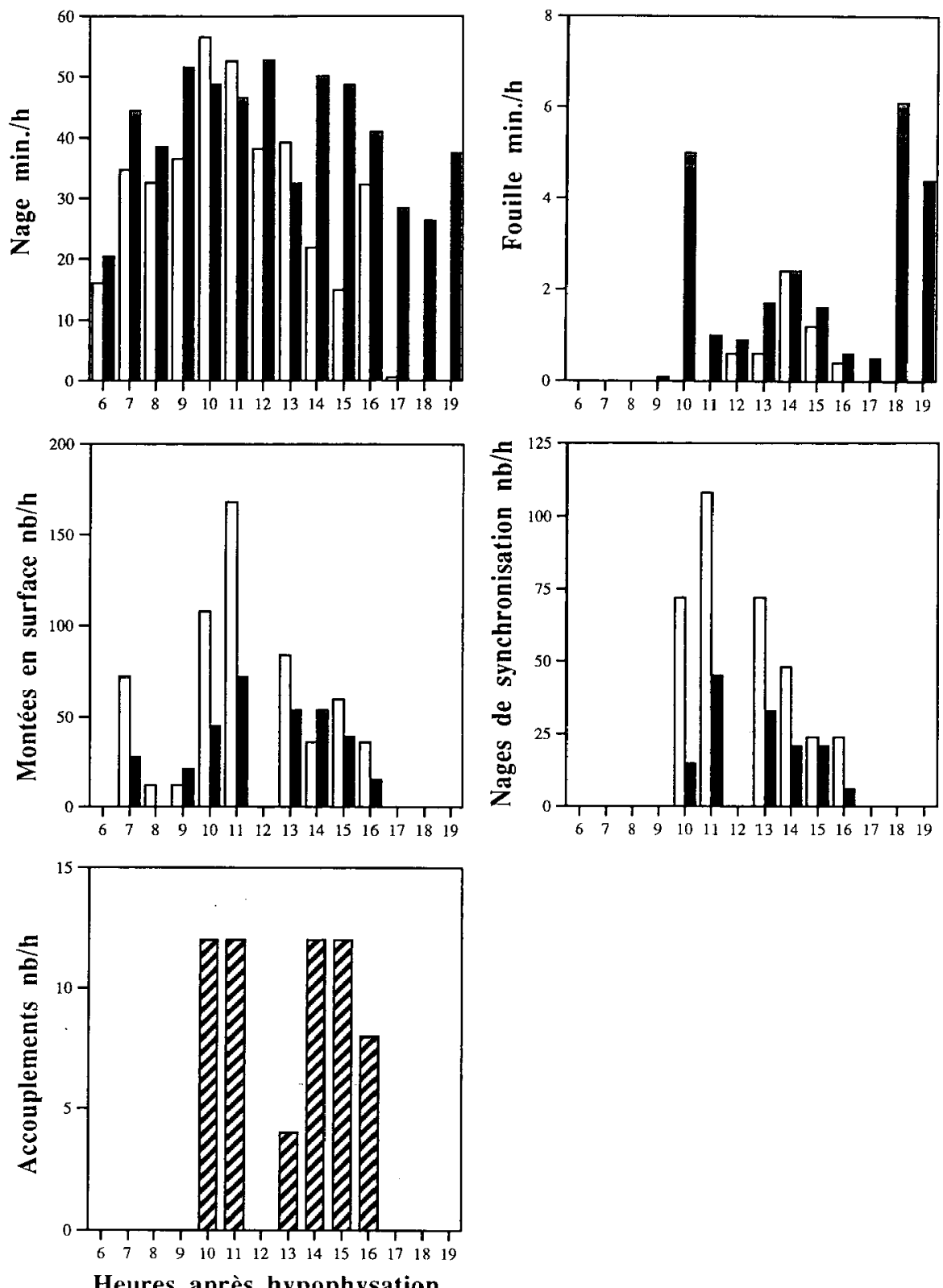

Figure 3

Principaux comportements (nage, fouille du gravier, montées en surface, nages de synchronisation, accouplements) du goujon au cours d'une journée de reproduction, après induction hormonale de la ponte dans un groupe composé de 3 femelles et 4 mâles. Seuls les résultats relatifs à la femelle qui a pondu (en blanc) et aux 4 mâles (en noir) ont été analysés, les deux autres femelles ne participant jamais aux accouplements. La fréquence des accouplements concerne à la fois les mâles et la femelle.

Figure 3

Main behaviours in the gudgeon (swimming activity, foraging, surfacing, synchronizing, spawning acts) during a spawning day, after hormonal treatment in a group of 3 females and 4 males. The results of the spawning female (in white) and of the 4 males (in black) were only analysed, because the two other females never exhibited reproductive behaviours. The spawning frequency concerns both males and the female. 
il est difficile d'estimer l'influence de l'induction hormonale sur l'expression des comportements. Toutefois, chez d'autres espèces de cyprinidés d'Europe, comme Barbus barbus, la description du comportement sexuel après hypophysation des géniteurs correspond parfaitement à celle établie avecc des poissons non hypophysés (JEANDARME et al., 1992). Cette constatation permet certainement de minimiser l'influence du processus d'induction hormonale de la ponte, utilisé aussi chez d'autres espèces de poissons (WOYNAROVICH et HORVATH, 1981). II est difficile d'expliquer le faible taux de reproduction des géniteurs. Une maturité insuffisante ou un stress lié à l'acclimatation en aquarium pourraient en être la cause. On peut aussi évoquer le caractère nocturne de l'activité (surtout alimentaire) du goujon. Toutefois, d'autres espèces de poissons cyprinidés comme Barbus barbus, dont les comportements sont principalement nocturnes, voient leur cycle d'activité s'inverser au moment de la reproduction : les accouplements se déroulent principalement de jour (BARAS, 1992).

Des pontes massives de goujons sur des substrats naturels (graviers) ou synthétiques (géotextile nylon) disposés dans des bassins d'élevage ont été obtenues par KESTEMONT et MELARD (1994), après injections hormonales des géniteurs. Ces auteurs ont mis en évidence l'importance du courant d'eau dans le choix du substrat. Dans le cadre de cette étude, bien qu'un courant d'eau était créé dans l'aquarium, il est apparu que les goujons développaient des comportements dans lesquels les reproducteurs accélèrent leur nage en direction de la surface de l'eau, indépendamment du courant créé. Ce comportement a pour effet de libérer les œufs dans la colonne d'eau. Ceux-ci peuvent alors être entrainés par le courant et se déposer dans les interstices du gravier ou sur un quelconque substrat dont, d'après nos observations, la nature ne semble pas intervenir dans l'expression du comportement sexuel. Ce dernier est quelque peu différent de celui des autres poissons d'eau vive comme les salmonidés (Salmo sp.) (JONES et BALL, 1954 ; FABRICIUS et GUSTAFSON, 1955) ou certains cyprinidés (Barbus barbus, Chondrostoma nasus) (LELEK et PENAZ, 1963 ; PONCIN, 1994) qui enfouissent activement leurs ovules dans le gravier. II se rapproche plus de celui de certains cyprins d'eau calme (Abramis brama) (PONCIN et al., 1996), d'ésocidés (Esox lucius) (FABRICIUS et GUSTAFSON, 1958) ou encore de clupéidés (Alosa alosa) (BOISNEAU et al., 1990) qui projettent leurs œufs dans la colonne d'eau, ceux-ci adhérant par la suite à la végétation aquatique ou se déposant sur un substrat quelconque. Si nos résultats préliminaires sur le goujon tendaient à se confirmer, il deviendrait intéressant de discuter du caractère psammophile de la reproduction de cette espèce.

Le pourcentage élevé de mortalité des œufs produits, causé par la prédation directe et importante (résultant probablement des conditions de confinement en aquarium) des géniteurs, ne nous a pas permis d'évaluer le succès des pontes. Une perturbation des géniteurs après une tentative de frai aurait été nécessaire pour aborder ce problème. Ceci n'a pas été réalisé, la priorité étant donnée aux observations comportementales.

En conclusion, ces résultats sur le comportement reproducteur du goujon reposent sur des observations précises (visualisation des ovules émis, notamment) qui ne pouvaient être réalisées que dans les conditions restreintes d'un aquarium et avec un petit nombre d'individus. Ces connaissances maintenant acquises, une étape ultérieure devrait consister à réaliser de telles observations sur un nombre plus important de poissons, dans les conditions semi-naturelles d'un fluvarium (rivière artificielle) par exemple, dispositif expérimental récemment opérationnel dans notre laboratoire. L'analyse des relations entre le comportement sexuel et la réalisation de pontes fractionnées chez le goujon (MANN, 1980) pourrait aussi être au centre de nos préoccupations.

Ces recherches ont bénéficié du support financier du Fonds National de la Recherche Scientifique (FNRS, crédits $n^{\circ} 9.4584 .91$ et 2.4525.94) et de l'aide technique de S. HOUBART, P. HUMPERS et J.M. BERTHOLET. Elles ont été réalisées sous la direction du Professeur J.C. RUWET. 


\section{BIBLIOGRAPHIE}

BALON E.K., 1975. Reproductive guilds of fishes : a proposal and a definition. J. Fish. Res. Board Can., 32, 821-864.

BARAS E., 1992. Etude des stratégies d'occupation du temps et de l'espace chez le barbeau fluviatile, Barbus barbus (L.). Cah. Ethol., 12, 125-442.

BOISNEAU P., MENNESSON-BOISNEAU C., BAGLINIĖRE J.L., 1990. Description d'une frayère et comportement de reproduction de la grande alose (Alosa alosa L.) dans le cours supérieur de la Loire. Bull. Fr. Pêche Piscic., 316, 15-23.

FABRICIUS E., GUSTAFSON K.J., 1955. Observation on the spawning behaviour of the grayling, Thymallus thymallus (L.). Report of the Institute of Freshwater Research, Drottningholm, 36, 75-103.

FABRICIUS E., GUSTAFSON K.J., 1958. Some new observations on the spawning behaviour of the pike, Esox lucius L. Report of the Institute of Freshwater Research, Drottningholm, 39, 23-54.

HOESTLAND H., BRUNET R., 1973. Le goujon, biologie et pisciculture. La pisciculture française d'eau vive et d'étang, 35, 31-34.

JEANDARME J., PONCIN P., BERREBI P., 1992. Etude préliminaire du comportement d'hybridation de Barbus barbus (L.) et de Barbus meridionalis (Risso) en aquarium. Cah. Ethol., 12, 519-528.

JONES J.W., BALL J.N., 1954. The spawning behaviour of brown trout and salmon. Brit. Journ. Anim. Behaviour, 2, 103-114.

KESTEMONT P., 1987. Etude du cycle reproducteur du goujon, Gobio gobio, L. 1. Variations saisonnières dans l'histologie de l'ovaire. J. Appl. Ichthyol., 3, 145-157.

KESTEMONT P., 1988 a. Etude des potentialités d'élevage intensif du goujon, Gobio gobio, L. Reproduction et croissance larvaire. Arch. et Micro Ed., Mus. Nat. Hist. Nat., Sect. Sc., Paris, 233 p.

KESTEMONT P., 1988 b. Effect of hormonal treatments on induced ovulation in gudgeon, Gobio gobio L. Aquaculture, 68, 373-385.

KESTEMONT P., 1989. Etude du cycle reproducteur des goujons, Gobio gobio, L. 2. Variations saisonnières dans l'histologie des testicules. J. Appl. Ichthyol., 5, 111-121.

KESTEMONT P., 1990. Dynamic aspects of ovogenesis in an asynchronous fish, the gudgeon Gobio gobio L. (Teleostei, Cyprinidae), under controlled temperature and photoperiod conditions. Aquat. Living Resour., 3, 61-74.

KESTEMONT P., MELARD C., 1994. L'élevage intensif du goujon et de quelques autres poissons d'eau douce (vairon, poisson rouge et ide mélanote). Ministère de la région wallonne de Belgique, Ed. Duculot, Belgique, $64 \mathrm{p}$.

LELEK A., PENAZ M., 1963. Spawning of Chondrostoma nasus (L.) in the Brumovka river. Zoologicke Listy, Folia Zoologica Brno, 12 (2), 121-134.

LOBON-CERVIA J., TORRES S., 1984. On the growth and reproduction of two populations of gudgeon (Gobio gobio L.) in Central Spain. Acta Hydrobiol., 25-26/1, 101-115.

MANN R., 1980. The growth and reproductive strategy of the gudgeon, Gobio gobio (L.), in two hard-water rivers in southern England. J. Fish Biol., 17, 163-176.

PENAZ M., PROKES M., 1978. Reproduction and early development of the gudgeon, Gobio gobio. I. Spawning and embryonic period. Folia Zoologica, 27, 257-267.

PHILIPPART J.C., 1989. Ecologie des populations de poissons et caractéristiques physiques et chimiques des rivières dans le bassin de la Meuse belge. Bull. Soc. Géographique Liège, 25, 175-198. 
PONCIN P., 1994. Bilan de 4 années d'étude du comportement reproducteur et de l'hybridation chez Barbus barbus (L., 1758) et Barbus meridionalis (Risso, 1826). Bull. Fr. Pêche Piscic., 334, 169-176.

PONCIN P., 1996. Reproduction chez nos poissons. Fédération Sportive des Pêcheurs Francophones de Belgique Ed., Nelles Imprimeries Havaux, Nivelles, 96 p.

PONCIN P., COMPERE P., CHRISTIAENS T., GEORGES M., BERREBI P., 1995. Spawning stimuli and paternity analysis in the European barbels. In Abstracts book of the International Round Table Barbus III, Liblice, Czech Republic, page 17.

PONCIN P., PHILIPPART J.C., RUWET J.C., 1996. Territorial and non-territorial spawning behaviour in the bream. J. Fish Biol., 49, 622-626.

PONCIN P., RUWET J.C., 1996. Le laboratoire d'Ethologie des Poissons rénové. Cah. Ethol., 16, 377-390.

TURNER G., 1986. Teleost mating systems and strategies. In The Behaviour of Teleost Fishes, Pitcher T. Ed., 253-274, Croom Helm, London.

WOYNAROVICH E., HORVATH L., 1981. La reproduction artificielle des poissons en eau chaude : manuel de vulgarisation. FAO Doc. Tech. Pêches, 201, 191 p. 